\title{
The Risk Assessment of Oxidative Stress in Postpartum Dairy Cattle
}

\author{
Yu Hongyi, Zhang Cuiyu, Qian Weidong, Zhao Chang, Zhang Hongyou \& Xia Cheng
}

\begin{abstract}
Background: Negative energy balance in cows will induce catabolism especially of lipids and the resulting rapid increase of free fatty acids (NEFA) in the blood stream increases the production of reactive oxygen metabolites (ROMs) and promote oxidative stress. Once the animal body is in an oxidative stress state, many molecules in the body may be peroxidized, accelerating the destruction of cells and causing damage to tissues or organs. This study conducted risk assessment of the oxidative stress caused by negative postpartum energy balance in cows by exploring the relationship between negative energy balance and oxidative stress.

Materials, Methods \& Results: This experiment randomly selected 120 cows at 14 to 21 days postpartum, from an intensive cattle farm in Heilongjiang province, China. Using a negative energy balance standard of $\beta$-hydroxybutyric acid (BHBA) greater than $1.2 \mathrm{mmol} / \mathrm{L}$, nonesterified fatty acid (NEFA) greater than 0.4 $\mathrm{mmol} / \mathrm{L}$ and glucose (GLU) less than three $\mathrm{mmol} / \mathrm{L}$, the cattle were divided into a healthy group of 74 cows and a diseased group of 46 cows. The oxidative stress indices of the experimental cows were measured and analyzed using the independent sample $t$ test. Spearman correlation analysis and regression analysis were performed and by using the binary Logistic regression analysis to predict disease, the receiver-operating characteristic curve (ROC) analysis established diagnosis effect and boundary values. Compared with the healthy group, the levels of glutathione peroxidase (GSH-Px), catalase (CAT), vitamin E (VE), selenium (Se) and total nitric oxide synthetase (T-NOS) in the blood of dairy cows in the diseased group were significantly reduced and the level of malondialdehyde (MDA) was significantly increased. The study concluded that negative energy balance is associated with oxidative stress in cows and the blood levels of GSH-Px, CAT and Se can be used to evaluate the degree of resulting oxidative stress. The early warning levels were determined to be GSH-Px less than 619.28 U/L, CAT less than $7.87 \mathrm{U} / \mathrm{ml}$ and Se less than $0.51 \mu \mathrm{g} / \mathrm{L}$.

Discussion: The levels of GSH-Px, CAT, Se, T-NOS and VE in the blood of cows in the disease group were lower than those in the healthy control group. This may be due to the increase in energy demand of cows in the perinatal period and the decrease of DMI in this period, which promotes the NEB of cows. In order to alleviate the NEB, the body forces its metabolic enhancement. Some tissues of the body cannot adjust their metabolism to adapt to NEB and this leads to excessive NEFA, which causes oxidative stress. Excessive NEFA can also affect the expression of GSH-Px in cells, and causing a series of oxidation reactions, increased free radicals and as the antioxidant system is overwhelmed. The MDA content in the blood of the deficient group was significantly increased compared with the healthy group, positively correlated with NEFA and BHBA and negatively correlated with GLU. This may be due to severe oxidative stress in cows during the period of negative energy balance as MDA is one of the important products of membrane lipid peroxidation. Long chain PUFA are an important component of all cell membranes and their oxidation damages the integrity, fluidity and function of cell membranes. In summary MDA causes oxidative damage to cells, damages mitochondrial membranes and changes membrane permeability, resulting in more free radicals produced by mitochondria during biological oxidation.
\end{abstract}

Keywords: dairy cows, negative energy balance, oxidative stress, ROC analysis, risk assessment. 


\section{INTRODUCTION}

The perinatal period is an important period for dairy cows. The body needs to consume a large amount of nutrients, but due to the prenatal and fetal pressure on the rumen and the influence of hormones, dairy cows' dry matter intake (DMI) decreases significantly [16]. The dry matter intake is lower than the body's consumption and postpartum energy intake accounts for only $80 \%$ of the requirement, leaving the body in a negative energy balance (NEB) state [5-7,17]. Negative energy balance in cows will induce catabolism and the resulting rapid increase of free fatty acids (NEFA) in the blood stream increases the production of reactive oxygen metabolites (ROMs) and promote oxidative stress $[2,15]$. There are two types of antioxidant system in animals including a non-enzymatic antioxidant system and an enzymatic antioxidant system. The function of both antioxidant systems is to protect the body from oxidative stress, but if the cows body generates too many ROMs, oxidative stress will result $[3,14]$.

Once the animal body is in an oxidative stress state, many molecules in the body may be peroxidized, accelerating the destruction of cells and causing damage to tissues or organs $[18,21]$. In addition, oxidative stress is one of the main factors that causes such diseases in dairy cows as ketosis, hepatic lipidosis, ovarian disease and hypocalcemia $[11,19]$.

This study conducted risk assessment of the oxidative stress caused by negative postpartum energy balance in cows by exploring the relationship between negative energy balance and oxidative stress and provides a new basis for early monitoring and early warning of oxidative stress in cows in the future.

\section{MATERIALS AND METHODS}

Test animals, sampling and grouping

This experiment was conducted on an intensive cattle farm in Heilongjiang Province, China. The cattle farm uses free feeding of total mixed rations (TMR) and has comprehensive cattle farm management software, which was used to randomly select 120 cows of similar age, 14 to 21 days post-partum and of similar condition score for this study. These cows had no abnormal clinical issues during the perinatal period and had a mean annual output of about nine tonnes per year.
These cows were divided into a diseased group of 46 cows with $\beta$-hydroxybutyric acid (BHBA) greater than $1.0 \mathrm{mmoI} / \mathrm{L}$, nonesterified fatty acids (NEFA) greater than $0.40 \mathrm{mmoI} / \mathrm{L}$ and glucose (GLU) less than 3.00 $\mathrm{mmoI} / \mathrm{L}$ and a healthy group of 74 cows with BHBA less than $1.20 \mathrm{mmol} / \mathrm{L}$, NEFA less than $0.40 \mathrm{mmol} / \mathrm{L}$ and GLU greater than $3.00 \mathrm{mmoI} / \mathrm{L}$. The prevalence rate of negative energy balance after delivery was $38.33 \%$.

Ten $\mathrm{mL}$ of tail base venous blood was taken before feeding from 14 to 21 days post-delivery and the serum was separated by centrifugation at $5000 \mathrm{~g}$ for 5 min. The serum was separated into EP tubes containing heparin ${ }^{1}$ and stored in a freezer at $-80^{\circ} \mathrm{C}$.

\section{Reagents and serum test methods}

Energy metabolism indices including GLU in $\mathrm{mmol} / \mathrm{L}$ and NEFA in $\mathrm{mmol} / \mathrm{L}$, both by colorimetric method and BHBA in mmol/L, by enzyme-linked immunoassay (ELISA) were measured in the Nanjing Jiancheng Bioengineering Institute ${ }^{2}$.

Oxidative stress indicators including superoxide dismutase (SOD), glutathione peroxidase (GSH$\mathrm{Px})$, catalase (CAT) and total nitric oxide synthetase (T-NOS) were all measured in U/mL by colorimetric method; malondialdehyde (MDA) in $\mathrm{nmol} / \mathrm{mL}$ by ELISA; vitamin C (VC) and vitamin E (VE) were all measured in nmol/L by ELISA and selenium $(\mathrm{Se})$ in $\mu \mathrm{g} / \mathrm{L}$ was determined by the atomic absorption method. These oxidative stress indicators were all measured in serum, using kits ${ }^{3}$ purchased from Shanghai Jinma biotechnology co., LTD.

\section{Analysis of data}

IBM SPSS (Statistics 19.0) software4 was used for biometric analysis of the experimental data and indicators that did not conform to normal distribution were selected by Kolmogorov-Smirnov (K-S) test for non-parametric Kruskal-Wallis (K-W) test. Independent sample $t$ test and Spearman correlation analysis were carried out for the indices conforming to the normal distribution, then binary logistic regression and ROC analyses were performed to determine the warning value.

\section{RESULTS}

As shown in Table 1, compared with the healthy group, the levels of GSH-Px, CAT, VE and Se in the blood of dairy cows in the diseased group were extremely significantly reduced, the level of T-NOS 
was significantly reduced and the level of MDA was significantly increased (Table 1).

Table 2 shows the correlation analysis of negative energy balance and oxidative stress. The levels of VE, GSH-Px, CAT and Se in blood were negatively correlated with BHBA, while the levels of MDA in blood was positively correlated with BHBA. The level of GSH-Px in blood showed a significant positive correlation with GLU, while the level of VE and MDA in blood showed a significant negative correlation with GLU. The levels of GSH-Px, CAT and VE were negatively correlated with NEFA (Table 2).

SPSS software was used to establish two binary logistic regression models to simulate the prediction of oxidative stress caused by negative energy balance of cows. Model I include GSH-Px, CAT, T-NOS and SOD; model II includes VE, VC, Se and MDA.

Table 3 and Figure 1 show the boundary value, sensitivity, specificity, standard error, positive likelihood ratio and area under the ROC curve. The optimal boundary values were determined as plasma GSH-Px less than 619.28 U/L, CAT less than 7.87 $\mathrm{U} / \mathrm{mL}$, and Se less than $0.51 \mu \mathrm{g} / \mathrm{L}$ by the Youden index. The corresponding sensitivities of GSH-Px, CAT and Se were 78.3, 73.9 and 95.7 respectively and the specificities were $69.7,91.7$ and 66.7 respectively. The area under the curve was $0.739,0.786$ and 0.828 respectively (Table 3 ). If the area under the ROC curve is greater than 0.5 , it has diagnostic significance (Figure 1).

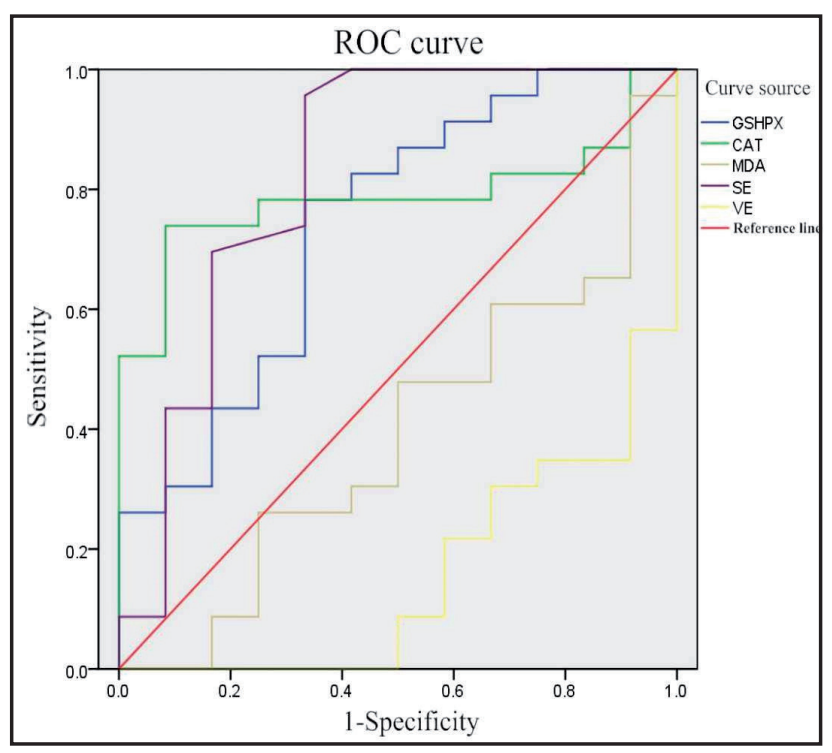

Figure 1. GSH-Px= glutathione peroxidase $\mathrm{CAT}=$ catalase $\mathrm{MDA}=$ malondialdehyde; $\mathrm{Se}=$ selenium; $\mathrm{VE}=$ vitamin $\mathrm{E}$.
Table 1. Comparison of background information and oxidative stress index of cows.

\begin{tabular}{ccc}
\hline Indicator & Morbidity group & Health group \\
\hline Number of cattle $(\mathrm{n})$ & 46 & 74 \\
Months of age & $40.72 \pm 0.67$ & $40.63 \pm 0.75$ \\
Parity & $1.73 \pm 0.45$ & $1.96 \pm 0.37$ \\
BSC & $3.14 \pm 0.18$ & $3.31 \pm 0.33$ \\
Milk production & $27.31 \pm 7.58$ & $25.17 \pm 4.37$ \\
SOD $(\mathrm{U} / \mathrm{mL})$ & $112.69 \pm 18.34$ & $109.33 \pm 12.91$ \\
GSH-Px $(\mathrm{U} / \mathrm{L})$ & $604.66 \pm 189.94^{\mathrm{A}}$ & $756.96 \pm 179.43^{\mathrm{B}}$ \\
CAT $(\mathrm{U} / \mathrm{mL})$ & $6.94 \pm 2.25^{\mathrm{A}}$ & $8.58 \pm 1.71^{\mathrm{B}}$ \\
T-NOS $(\mathrm{U} / \mathrm{mL})$ & $41.46 \pm 6.48^{\mathrm{a}}$ & $44.69 \pm 5.71^{\mathrm{b}}$ \\
VC $(\mathrm{nmol} / \mathrm{L})$ & $3340.33 \pm 473.66$ & $3294.41 \pm 351.58$ \\
VE $(\mathrm{nmol} / \mathrm{L})$ & $523.05 \pm 55.58^{\mathrm{A}}$ & $601.65 \pm 95.97^{\mathrm{B}}$ \\
Se $(\mu \mathrm{g} / \mathrm{L})$ & $0.51 \pm 0.06^{\mathrm{A}}$ & $0.57 \pm 0.04^{\mathrm{B}}$ \\
MDA $(\mathrm{nmol} / \mathrm{mL})$ & $15.79 \pm 2.10^{\mathrm{a}}$ & $14.63 \pm 1.53^{\mathrm{b}}$ \\
\hline
\end{tabular}

Different uppercase letters in the table indicate $P<0.01$ and different lowercase letters indicate $P<0.05$. The same letter or no letter indicates that the difference is not significant $P>0.05$. BCS= body condition score; $\mathrm{SOD}=$ superoxide dismutase; GSH-Px=glutathione peroxidase; $\mathrm{CAT}=$ catalase; $\mathrm{T}-\mathrm{NOS}=$ total nitric oxide synthetase; $\mathrm{VC}=$ vitamin $\mathrm{C} ; \mathrm{VE}=$ vitamin $\mathrm{E} ; \mathrm{Se}=$ selenium; MDA= malondialdehyde.

\section{DISCUSSION}

Negative energy balance of post parturient cows is a problem affecting most countries with a cattle industry and is caused by high energy output and low dry matter intake [13]. It can cause many secondary diseases of cows, such as ketosis, fatty liver, dystocia and infectious diseases, potentially causing significant economic losses to the farmer [1]. At the same time, in order to adapt to the negative energy balance, cows use their own fat reserves to balance the transformation and physiological metabolism of energy substances, because the liver and other tissues find it difficult to maintain homeostasis and this greatly increases the probability of oxidative stress in the body [10]. The purpose of this study was to analyze the correlation between the oxidative stress index and negative energy balance and to select specific factors to assess the risk of oxidative stress caused by negative energy balance in cows.

As shown in Table 1 of the results, the levels of GSH-Px, CAT, Se, T-NOS and VE in the blood of cows in the disease group were lower than those in the healthy control group. This may be due to the increase in energy demand of cows in the perinatal period and the decrease of DMI in this period, which promotes the NEB of cows. In order to alleviate the NEB, the body forces its metabolic enhancement [9]. 
Table 2. Correlation between negative energy balance index and oxidative stress index.

\begin{tabular}{ccccccc}
\hline Indicator & \multicolumn{2}{c}{ Correlation with BHBA } & \multicolumn{2}{c}{ Correlation with GLU } & \multicolumn{2}{c}{ Correlation with NEFA } \\
\hline SOD $(\mathrm{U} / \mathrm{mL})$ & $\mathrm{R}=0.15$ & $P=0.31$ & $\mathrm{R}=-0.09$ & $P=0.51$ & $\mathrm{R}=0.00$ & $P=0.95$ \\
GSH $-P \mathrm{x}(\mathrm{U} / \mathrm{L})$ & $\mathrm{R}=-0.48^{* *}$ & $P=0.00$ & $\mathrm{R}=0.46^{* *}$ & $P=0.00$ & $\mathrm{R}=-0.29^{*}$ & $P=0.04$ \\
CAT $(\mathrm{U} / \mathrm{mL})$ & $\mathrm{R}=-0.35^{*}$ & $P=0.01$ & $\mathrm{R}=-0.02$ & $P=0.88$ & $\mathrm{R}=-0.41^{* *}$ & $P=0.00$ \\
$\mathrm{~T}-\mathrm{NOS}(\mathrm{U} / \mathrm{mL})$ & $\mathrm{R}=-0.26$ & $P=0.06$ & $\mathrm{R}=0.14$ & $P=0.34$ & $\mathrm{R}=-0.12$ & $P=0.40$ \\
$\mathrm{VC}(\mathrm{nmol} / \mathrm{L})$ & $\mathrm{R}=-0.07$ & $P=0.60$ & $\mathrm{R}=0.07$ & $P=0.60$ & $\mathrm{R}=-0.11$ & $P=0.44$ \\
$\mathrm{VE}(\mathrm{nmol} / \mathrm{L})$ & $\mathrm{R}=-0.37^{* *}$ & $P=0.00$ & $\mathrm{R}=-0.28^{*}$ & $P=0.04$ & $\mathrm{R}=-0.30^{*}$ & $P=0.03$ \\
$\mathrm{Se}(\mu \mathrm{g} / \mathrm{L})$ & $\mathrm{R}=-0.36^{*}$ & $P=0.01$ & $\mathrm{R}=0.08$ & $P=0.55$ & $\mathrm{R}=-0.84$ & $P=0.57$ \\
$\mathrm{MDA}(\mathrm{nmol} / \mathrm{mL})$ & $\mathrm{R}=0.23^{*}$ & $P=0.03$ & $\mathrm{R}=-0.16^{*}$ & $P=0.25$ & $\mathrm{R}=0.36^{*}$ & $P=0.01$ \\
\hline
\end{tabular}

$* * P<0.01 ; * P<0.05$; and absence of $*$ indicates that the difference is not significant $(P>0.05)$. SOD $=$ suPeroxide dismutase; GSH- $P \mathrm{x}=$ glutathione $P$ eroxidase $; \mathrm{CAT}=$ catalase; $\mathrm{T}-\mathrm{NOS}=$ total nitric oxide synthetase $\mathrm{VC}=$ vitamin $\mathrm{C} ; \mathrm{VE}=$ vitamin $\mathrm{E} ; \mathrm{Se}=$ selenium; $\mathrm{MDA}=$ malondialdehyde.

Table 3. Cut-off value, Sensitivity, Specificity, Negative, Standard Error, Positive Still Ratio, and the Area under the ROC Curve.

\begin{tabular}{cccccccc}
\hline Indicator & cut-off value & Sensitivity $(\%)$ & Specificity $(\%)$ & SE & +LR & AUC \\
\hline GSH-Px & 619.28 & 78.3 & 73.9 & 69.7 & 0.091 & 2.35 & 0.739 \\
CAT & 7.87 & 65.4 & 91.7 & 0.079 & 8.90 & 0.786 \\
MDA & 12.72 & 95.7 & 70.3 & 0.102 & 1.04 & $P=0.006$ \\
Se & 0.51 & 75.7 & 66.7 & 0.086 & 2.87 & 0.391 & 0.828 \\
Ve & 437.12 & 53.2 & 0.068 & 0.96 & 0.156 & $P=0.001$ \\
\hline
\end{tabular}

$\mathrm{SE}=$ standard error; $+\mathrm{LR}=$ Positive likelihood ratio; $\mathrm{AUC}=$ area under curve.

Some tissues of the body cannot adjust their metabolism to adapt to NEB [8] and this leads to excessive NEFA, which causes oxidative stress [4]. Excessive NEFA can also affect the expression of GSH-Px in cells, and causing a series of oxidation reactions, increased free radicals and as the antioxidant system is overwhelmed, this leads to the elimination of free radicals far less rapidly than the generation of free radicals, so the cattle blood GSH-Px and CAT, Se, VE, T-NOS will be reduced.

The MDA content in the blood of the deficient group was significantly increased compared with the healthy group, positively correlated with NEFA and BHBA and negatively correlated with GLU. This may be due to severe oxidative stress in cows during the period of negative energy balance as MDA is one of the important products of membrane lipid peroxidation. In the event of oxidative stress, more free radicals will be produced, which will act on lipids causing peroxidation. The increase of MDA content in the blood of cows in the disease group was consistent with the results reported in other literature [20] and it can oxidize polyunsaturated fatty acids (PUFA) in cells. Long chain PUFA are an important component of all cell membranes and their oxidation damages the integrity, fluidity and function of cell membranes. In summary MDA causes oxidative damage to cells, damages mitochondrial membranes and changes membrane permeability, resulting in more free radicals produced by mitochondria during biological oxidation [12]. In this study, ROC analysis was used to define the early warning values of three indexes in blood by binary logistic regression. When GSH-Px $<619.28 \mathrm{U} / \mathrm{L}$, $\mathrm{CAT}<7.87 \mathrm{U} / \mathrm{mL}, \mathrm{Se}<0.51 \mu \mathrm{g} / \mathrm{L}$, the body is prone to oxidative stress.

\section{CONCLUSIONS}

This study confirmed that negative energy balance is one of the important factors causing postpartum oxidative stress in dairy cows and it determined three levels of GSH-Px, CAT and Se as early warning indices of oxidative stress caused by negative energy balance, laying a theoretical foundation for more effective monitoring and prevention of negative energy balance and oxidative stress on cattle farms in future.

\section{MANUFACTURERS}

${ }^{1}$ Saiguo biotech Co. Ltd. Guangzhou, China.

${ }^{2}$ Nanjing Jiancheng Bioengineering Institute. Nanjing, China

${ }^{3}$ Jinma biotechnology. Co. Ltd. Shanghai, China.

${ }^{4}$ IBM. New York, NY, USA. 
Funding. This study was funded by the following projects: Screening and confirmation of serum biomarkers for subclinical vitamin E deficiency in early lactating high-yield dairy cows (31873028), research on prevention and control technology of common diseases of livestock and poultry (2017YFD0502200), demonstration project of poverty alleviation in standardized breeding industry (ZY17C08).

Acknowledgments. We thank International Science Editing (http://www.internationalscienceediting.com) for editing this manuscript.
Ethical approval. The study protocol was approved by the Ethics Committee on the Use and Care of Animals of Heilongjiang Bayi Agricultural University (Daqing, China). The study was approved by the farm owner and all animal experiments were conducted according to the International Guiding Principles for Biomedical Research. The protocol was approved by the Committee on the Ethics of Animal Experiments of the Heilongjiang Bayi Agricultural University.

Declaration of interest. The authors report no conflicts of interest. The authors alone are responsible for the content and writing of paper.

\section{REFERENCES}

1 Butler W.R. \& Smith R.D. 1989. Interrelationships between energy balance and postpartum reproductive function in dairy cattle. Journal of Dairy Science. 72(3): 767-783.

2 Bernabucci U., Ronchi B., Lacetera N. \& Nardone A. 2005. Influence of body condition score on relationships between metabolic status and oxidative stress in periparturient dairy cows. Journal of Dairy Science. 88(6): 2017-2026.

3 Brzezinska-Slebodzinska E., Miller J.K., Quigley III J.D., Moore J.R. \& Madsen F.C. 1994. Antioxidant status of dairy cows supplemented prepartum with vitamin E and selenium. Journal of Dairy Science. 77(10): 3087-3095.

4 Castillo C., Hernandez J., Bravo A., Lopez-Alonso M., Pereira V. \& Benedito J.L. 2005. Oxidative status during late pregnancy and early lactation in dairy cows. The Veterinary Journal. 169(2): 286-292.

5 Drackley J.K. 1999. Biology of dairy cows during the transition period: The final frontier. Journal of Dairy Science. 82(11): 2259-2273.

6 Esposito G., Irons P.C., Webb E.C. \& Chapwanya A. 2014. Interactions between negative energy balance, metabolic diseases, uterine health, and immune response in transition dairy cows. Animal Reproduction Science. 144(3-4): 60-71.

7 Grummer R.R. 1995. Impact of changes in organic nutrient metabolism on feeding the transition dairy cow. Journal of Animal Science. 73(9): 2820-2833.

8 Guo J., Peters R.R. \& Kohn R.A. 2007. Effect of a transition diet on production performance and metabolism in periparturient dairy cows. Journal of Dairy Science. 90(11): 5247-5258.

9 Gross J., van Dorland H.A., Bruckmaier R.M. \& Schwarz F.J. 2011. Performance and metabolic profile of dairy cows during a lactational and deliberately induced negative energy balance with subsequent realimentation. Journal of Dairy Science. 94(4): 1820-1830.

10 Herdt T.H. 2000. Ruminant adaptation to negative energy balance: Influences on the etiology of ketosis and fatty liver. Veterinary Clinics: Food Animal Practice. 16(2): 215-230.

11 Higdon J.V. \& Frei B. 2003. Obesity, and oxidative stress: a direct link to CVD? Arteriosclerosis, Thrombosis, and Vascular Biology. 23(3): 365-367.

12 Konvičná J., Vargová M., Paulíková I., Kováč G. \& Kostecká Z. 2015. Oxidative stress and antioxidant status in dairy cows during prepartal and postpartal periods. Acta Veterinaria Brno. 84(2): 133-140.

13 Mozduri Z., Bakhtiarizadeh M.R. \& Salehi A. 2018. Integrated regulatory network reveals novel candidate regulators in the development of negative energy balance in cattle. Animal: An International Journal of Animal Bioscience. 12(6): 1196-1207.

14 Miller J.K., Brzezinska-Slebodzinska E. \& Madsen F.C. 1993. Oxidative stress, antioxidants, and animal function. Journal of Dairy Science. 76(9): 2812-2823.

15 Nordberg J. \& Arnér E.S. 2001. Reactive oxygen species, antioxidants, and the mammalian thioredoxin system. Free Radical Biology and Medicine. 31(11): 1287-1312.

16 Overton T.R. \& Waldron M.R. 2004. Nutritional management of transition dairy cows: strategies to optimize metabolic health. Journal of Dairy Science. 87: E105-E119.

17 Rosendo O., Staples C.R., McDowell L.R., McMahon R., Badinga L., Martin F.G., Shearer J.F., Seymour W.M. \& Wilkinson N.S. 2004. Effects of biotin supplementation on peripartum performance and metabolites of Holstein cows. Journal of Dairy Science. 87(8): 2535-2545.

18 Sies H. 2015. Oxidative stress: a concept in redox biology and medicine. Redox Biology. 4: 180-183. 
19 Sordillo L.M. \& Aitken S.L. 2009. Impact of oxidative stress on the health and immune function of dairy cattle. Veterinary Immunology and Immunopathology. 128(1-3): 104-109.

20 Turk R., Juretić D., Gereš D., Svetina A., Turk N. \& Flegar-Meštrić Z. 2008. Influence of oxidative stress and metabolic adaptation on PON1 activity and MDA level in transition dairy cows. Animal Reproduction Science. 108(12): 98-106.

21 Turk R., Juretic D., Geres D., Turk N., Rekic B., Simeon-Rudolf V. \& Svetina A. 2004. Serum paraoxonase activity and lipid parameters in the early postpartum period of dairy cows. Research in Veterinary Science. 76(1): 57-61. 